\section{VTE PROPHYLAXIS IN A PALLIATIVE CARE INPATIENT UNIT AND BEYOND: GETTING OUR MESSAGE STRAIGHT}

Angela C Star, Hayley Evans, Bethany Clark, Eleanor Smith. Sheffield Teaching Hospitals NHS Foundation Trust

10.1136/bmjspcare-2019-ASP.63

Background Recently published evidence $^{1}$ highlighted high bleeding risks in palliative care (PC) cohorts. Evidence supporting venous thromboembolism prophylaxis (VTEP) generally comes from acute medicine or oncology.

Local trust guidelines mandated urgent VTEP assessment, recommending VTEP for most medical inpatients. No local guidance existed for PC. Anecdotally, practice within the attached specialist PC inpatient unit (IPU) varied.

Aims To audit VTEP assessment and administration against trust and NICE guidelines.

Methods Trust audit approval was obtained. Electronic and paper notes were examined for IPU stays between May-Aug 2017. Results were anonymised, using Excel for analysis.

Results Notes were available for 86/96 identified patient episodes. Thirteen patients receiving anticoagulation treatment were excluded, leaving $\mathrm{n}=73$ episodes for analysis (including repeat stays). Most patients were elderly and had cancer. $86 \%$ of episodes had recorded VTEP initial assessments; 100\% had VTEP prescribed (or a documented clinical reason), accordingly. Only 6/7 patients with potential VTEP complications had their VTEP re-assessed.

$73 \%$ episodes included terminal care. Where death was unexpected $(n=48)$, most patients stopped receiving VTEP either when dying was diagnosed $(n=31)$ or at another time before death $(n=8)$. This was not always a formal medical decision.

Actions Results were presented at the PC audit meeting. A departmental VTEP policy was drafted, which provided input to the trust thrombosis committee and future trust-wide policy. Holistic assessment of VTEP appropriateness and re-assessment at key clinical points were emphasised through teaching. IPU consultant ward-round stickers were created, encouraging formal re-assessment. Re-audit 2018 confirmed widespread improvement of targets.

Conclusions Variation in VTEP practice reflected lack of clarity about PC in the trust's policy. Changes to departmental policy and engagement with trust policymakers helped effectively align practice with NICE guidance, prioritising patientcentric care, shared decision-making and minimising potentially harmful medications.

\section{REFERENCE}

1. Tardy B, et al. The RHESO study 2017.

\section{DESCRIBING THE END OF LIFE EXPERIENCE OF PATIENTS SUPPORTED WITH A LIFE-SUSTAINING LEFT VENTRICULAR ASSIST DEVICE (LVAD) AS BRIDGE TO TRANSPLANTATION IN THE UK - CAN WE HELP?}

Donna Wakefield, Maria McKenna. Freeman Hospital, Newcastle-Upon-Tyne Hospitals NHS Foundation Trust

\subsection{6/bmispcare-2019-ASP.64}

Background In the UK, patients with severe heart failure suitable for heart transplantation may be managed with a life-sustaining LVAD as a bridge to transplant. Many will not undergo transplantation, due to lack of donor organs or clinical deterioration rendering them unfit for surgery. These patients are at risk of LVAD-related complications and will die with their device in situ. We describe the end of life for this patient group.

Method Retrospective case note review of 40 deceased patients who underwent LVAD implantation between 2010 and 2016 at a single UK centre.

Results Within this cohort, mean age at death was 57 years, with a median interval between LVAD implantation and death of 18 months(range 6-76). Death was described as sudden in 17 patients; this group commonly died at home(65\%).

Serious LVAD-associated complications were seen in 35 patients (87.5\%), namely VAD thrombus (30\%), intracerebral bleed (30\%), GI bleed (27.5\%) or driveline infection $(25 \%)$. These complications were often fatal. This group had frequent (median 5, range 0-35) and lengthy (median 57 days, range 0-267) hospital admissions to their specialist centre, which for the majority $(87.5 \%)$ was not in their local area.

Where dying was identified and death expected $(n=19)$, patients were most likely to die in hospital (84\%), but transfer to local hospital $(5 \%)$ or hospice $(16 \%)$ was achieved for some. Anticipatory SC PRN medications were prescribed for $68 \%$ and symptom assessment undertaken in $84 \%$ of cases. Overall, preferred place of death was documented for 12 patients and achieved in 10/12 cases.

Conclusion The last days of life for patients with LVAD support, were commonly spent in hospital following active management of a LVAD-related complication. Early specialist palliative care input is recommended to improve quality of end of life care, support exploration of future care wishes and consideration of practical device management.

\section{THROMBOPROPHYLAXIS IN THE LAST DAYS OF LIFE}

Alice Martin, Emma Valentine. Torbay and South Devon NHS Foundation Trust

\subsection{6/bmjspcare-2019-ASP.65}

Background Venous thromboembolism (VTE) is a well-established cause of in-hospital mortality. Consequently, prevention is a vital part of patient care. Nevertheless, in patients approaching the end of life, VTE prophylaxis should be reviewed daily, with decision-making taking into account the views of the patient and carers. NICE advises VTE prophylaxis should not be offered to people in their last days of life. Methods We undertook a retrospective review of the casenotes of all patients who died at a district general hospital in the month 17th September - 17th October 2018.

We aimed to assess whether patients dying in the acute hospital were:

1. Commenced on VTE prophylaxis during admission;

2. Recognised as dying;

3. Commenced on the hospital's individualised care plan for the last days of life;

4. Receiving VTE prophylaxis in the 72 hours before death.

Results 96 patients died within the review period. 5 deaths in the emergency department were excluded. Of the remaining 91 , the median age was 82 .

47 out of the 91 patients (52\%) received VTE prophylaxis during their hospital admission.

68 patients $(75 \%)$ were recognised as dying, and of these, $40(59 \%)$ were commenced on the hospital's individualised care plan for the last days of life. 
25 patients received VTE prophylaxis within the last 72 hours of life. Of these, 12 had VTE prophylaxis stopped in the coming days. 6 patients who were recognised as dying received VTE prophylaxis in their last 24 hours of life.

Conclusions The results suggest that guidance regarding pharmacological thromboprophylaxis in the last days of life is not consistently followed even when the dying process has been recognised. They also highlight that improvement is needed in recognising the dying patient in the acute setting and that locally there is inadequate uptake of our 'last days of life' care plan.

\section{COMPARING THE ASSESSMENT OF END OF LIFE CARE IN THE STRUCTURED JUDGEMENT REVIEW WITH AN END OF LIFE AUDIT}

Sara Robbins, Kate Tredgett. Gloucestershire Hospitals NHS Foundation Trust

10.1136/bmjspcare-2019-ASP.66

Background The Structured Judgement Review (SJR) is a validated and standardised method for reviewing case notes of patients who died in hospital. One section assesses end of life care (EOLC); this can be rated from very poor to excellent. This audit aims to evaluate whether the SJR is providing us with an accurate assessment of the quality of EOLC in an Oncology inpatient department.

Methods This is a retrospective audit of adult patients that died on Oncology wards in 2017 and had an SJR completed. The end of life audit assessed whether the Hospital Trust's Key Performance Indicators (KPIs) were achieved. National standards were used to audit specific aspects of the individualised care plan. The care ratings and comments from each SJR were then compared with the end of life audit.

Results The notes of six patients were reviewed. The SJR ratings for EOLC were 'very good' in five cases and 'excellent' in one. However, the EOLC audit using Trust KPI's showed more variation in the quality of care. In three cases where the SJR rated 'very good' or 'excellent', this compared well with the EOLC audit where they achieved $80 \%-90 \%$ of Trust KPI's. In two cases where the SJR rated 'very good', they only achieved $30 \%$ of Trust KPI's. The final case was again rated 'very good', but only achieved $50 \%$ of Trust KPI's. The comments in the SJR did not discuss the same issues that the EOL audit raised. For example, some focused on treatment escalation plans being completed rather than the quality of care.

Conclusion The recorded quality of EOLC according to SJR's did not consistently reflect the quality of care being given. In order to improve the accuracy of the SJR, we attached key KPI's on EOLC to the SJR form as a prompt for reviewers.

\section{SERVICE EVALUATION OF THE USE OF FLUIDS AT END OF LIFE ACROSS TWO INPATIENT PALLIATIVE CARE UNITS}

Jenny Brocklebank, Constantina Pitsillides, Olivia Liddle, Roseleen Sheehan, Rachel Parry, Samuel Fingas, Eleanor Smith. Sheffield Teaching Hospitals, Macmillan Palliative Care Unit, St Luke's Hospice

10.1136/bmjspcare-2019-ASP.67
Background 'Five Priorities of Care' requires an individualised plan for hydration at end of life and discussion with patients/ those important to them. However there is little research surrounding this topic and the use of fluids is often dependent upon the individual prescriber.

Methods A service evaluation of the current practice in Sheffield's inpatient palliative care units.

A retrospective case-note review of deceased patients over a two-month period at St Luke's Hospice (SLH) and Macmillan Palliative Care Unit (MPCU), 37 and 20 cases respectively.

A qualitative staff survey of individual thoughts and practices with fluids.

Results Consistently good documentation of discussions about hydration risks and benefits, but less for symptoms of dry mouth and mouth care. These discussions were documented more often at SLH than MPCU (97/81\% vs 60/35\%). MPCU offered and prescribed fluids 2.5 times more often. Most commonly prescribed 1 litre of normal saline over 24 hours. Most common reason for stopping fluids was skin pooling $(75 \%$ SLH) and secretions (89\% MPCU). Documentation of discussions surrounding this seen for all cases SLH, 50\% cases MPCU.

An inability to quench thirst ranked first on survey regarding reasons for fluid delivery, opposed to dry mouth in clinical practice. Opinion was that the likely benefit from fluids was almost equal to the risk of harm. Subcutaneous delivery was the preferred route for being less invasive.

Conclusions Hydration at end of life needs to be individualised and regularly reviewed. Documentation was higher at SLH than MPCU regarding fluids, due to the ADD CARING pneumonic. Steps have been made at MPCU to prompt consideration of fluids at end of life. Improved documentation needed for dry mouth symptoms/mouth care. Staff responses did not always correspond to documented reasons for initiating and stopping fluids, suggesting these need to be decisions made across the MDT.

\section{ARCHEOLOGICAL DIG: A HISTORICAL REVIEW OF LOCAL EVIDENCE, GUIDELINES AND CLINICAL PRACTICE IN RESPONSE TO THE GOSPORT ENQUIRY}

Clare Finnegan, Karen E Groves. Lancashire Care Foundation Trust, Queenscourt Hospice, Southport and Ormskirk NHS Hospital Trust

\subsection{6/bmjspcare-2019-ASP.68}

Background The Gosport Report suggests over 450 patients died due to 'dangerous doses' of medication combinations without clinical indication. A cross-boundary integrated specialist palliative care service reviewed factors influencing prescribing culture for patients approaching end-of-life.

Methods A historical review of pain management and syringe driver use was considered alongside locality guidelines. Training programmes were reviewed and meetings held with stakeholders. Audits were undertaken to seek assurance of safe local prescribing practices.

Results

- During the Gosport period, there was clear guidance available for safe opioid starting doses, dose equivalences \& syringe driver use.

- Medical Devices Alerts regarding the risks of multiple syringe driver devices in use, led to use of a single device across locality. Local guidelines have always stated no indication for 'anticipatory prescribing for medication via a syringe driver'. 